INPLASY

PROTOCOL

To cite: Liu et al. Efficacy and safety of chloroquine and hydroxychloroquine in the treatment of patients with COVID-19 combined with diabetes mellitus a protocol for systematic review and metaanalysis. Inplasy protocol 202070109. doi:

10.37766/inplasy2020.7.0109

Received: 24 July 2020

Published: 24 July 2020

Corresponding author:

Yan Liu

liuxiaoyangas@163.com

Author Affiliation:

Affiliated Hospital/Clinical

School of Chengdu

University of Traditional

Chinese Medicine

Support: NSFC.

Review Stage at time of this submission: Preliminary searches.

Conflicts of interest: None.

\section{Efficacy and safety of chloroquine and hydroxychloroquine in the treatment of patients with COVID-19 combined with diabetes mellitus a protocol for systematic review and meta-analysis}

Liu, Y1; Fu, X²; Xie, $\mathrm{C}^{3}$.

Review question / Objective: Study design: Our research will include randomized controled trials. However, repeated publications of the same study; articles on research in pediatric populations (17 years of age or younger); letters, abstracts, reviews, or animal experiments are excluded. Participants: We will include all adult patients diagnosed with COVID-19. Interventions and comparators: The experimental group is patients diagnosed with COVID-19 and diabetes, while the control group is COVID-19 patients without diabetes. Both groups of patients received conventional COVID-19 treatment: antiviral, symptomatic and supportive treatment. The experimental group received conventional diabetes treatment recommended by the American Diabetes Association (ADA) guidelines, including diet, exercise, hypoglycemia and lipid-lowering treatment and chloroquine/ hydroxychloroquine treatment, and the control group received placebo or no treatment. Outcomes: The primary outcomes include Sputum virus nucleic acid negative time, lung imaging improvement time, mortality rate, mechanical ventilation rate, intensive care unit (ICU) hospitalization time, hospitalization time, clinical improvement, fasting blood glucose, 2-hour postprandial blood glucose, glycosylated hemoglobin, fasting insulin, symptoms Improvement, adverse reactions, etc.

INPLASY registration number: This protocol was registered with the International Platform of Registered Systematic Review and Meta-Analysis Protocols (INPLASY) on 24 July 2020 and was last updated on 24 July 2020 (registration number INPLASY202070109).

\section{INTRODUCTION}

Review question / Objective: Study design: Our research will include randomized controled trials. However, repeated publications of the same study; articles on research in pediatric populations (17 years of age or younger); letters, abstracts, reviews, or animal experiments are excluded. Participants: We will include all 
adult patients diagnosed with COVID-19. Interventions and comparators: The experimental group is patients diagnosed with COVID-19 and diabetes, while the control group is COVID-19 patients without diabetes. Both groups of patients received conventional COVID-19 treatment: antiviral, symptomatic and supportive treatment. The experimental group received conventional diabetes treatment recommended by the American Diabetes Association (ADA) guidelines, including diet, exercise, hypoglycemia and lipidlowering treatment and chloroquine/ hydroxychloroquine treatment, and the control group received placebo or no treatment. Outcomes: The primary outcomes include Sputum virus nucleic acid negative time, lung imaging improvement time, mortality rate, mechanical ventilation rate, intensive care unit (ICU) hospitalization time, hospitalization time, clinical improvement, fasting blood glucose, 2-hour postprandial blood glucose, glycosylated hemoglobin, fasting insulin, symptoms Improvement, adverse reactions, etc.

Condition being studied: Coronaviruses (CoVs) are enveloped single-stranded RNA viruses. Novel coronavirus pneumonia (COVID-19) has a higher prevalence and lower mortality rate than Severe Acute Respiratory Syndrome Coronavirus(SARSCoV). At the time of writing, COVID-19 has caused $15,727,530$ infections and 637,780 deaths in the worldwide. Diabetes mellitus (DM) is a common chronic metabolic disease, accompanied by multiple organs damage and functions decrease. COVID-19 is generally susceptible around the world, however, diabetic patients often have multiple chronic diseases and a declining immune response, which is a high-risk group susceptible to infect by this virus. According to the current published literatures, the proportion of diabetes in COVID-19 patients is $10.1 \%-20.0 \%$, and the proportion of diabetes in critically ill COVID-19 patients is $22.2 \%$. Patients with diabetes and COVID-19 may require special attention and clinical care. In the current global shortage of medical resources and lack of effective drugs, the low-cost antimalarial drugs chloroquine (CQ) and its derivative hydroxychloroquine (HCQ) have attracted more and more attention.

\section{METHODS}

Participant or population: We will include all adult patients diagnosed with COVID-19.

Intervention: Both groups of patients received conventional COVID-19 treatment. The experimental group received conventional diabetes treatment recommended by the American Diabetes Association (ADA) guidelines, including diet, exercise, hypoglycemia and lipidlowering treatment and chloroquine/ hydroxychloroquine treatment, and the control group received placebo or no treatment.

Comparator: The experimental group is patients diagnosed with COVID-19 and diabetes, while the control group is COVID-19 patients without diabetes.

Study designs to be included: Our research will include randomized controled trials.

Eligibility criteria: Our research will include randomized controled trials. However, repeated publications of the same study; articles on research in pediatric populations (17 years of age or younger); letters, abstracts, reviews, or animal experiments are excluded. We will include all adult patients diagnosed with COVID-19.This experiment involves chloroquine/hydroxychloroquine in treatment. Languages of the publications will be limited to English and Chinese.

Information sources: We will retrieve each database from December 2019 to July 2020. Chinese literature comes from CNKI, Wanfang, VIP, and CBM databases. English literature mainly searches Cochrane Library, PubMed, Web of Science, and EMBASE. We adopt the combination of heading terms and free words as search strategy, which decided by all the reviewers. Meanwhile, We will search other resources on the corresponding website to 
find the clinical trial registries and gray literature to complete the deficiencies of the electronic databases. Languages of the publications will be limited to English and Chinese.

Main outcome(s): The primary outcomes include Sputum virus nucleic acid negative time, lung imaging improvement time, mortality rate, mechanical ventilation rate, intensive care unit (ICU) hospitalization time, hospitalization time, clinical improvement, fasting blood glucose, 2hour postprandial blood glucose, glycosylated hemoglobin, fasting insulin, symptoms Improvement, adverse reactions, etc.

Quality assessment / Risk of bias analysis: Two independent reviewers will evaluate the included literature on the risk of bias according to the guidelines of the Cochrane Handbook for Systematic Reviews of Interventions. The evaluation items are: random sequence generation, allocation concealment, blinding participants and personnel, blinding evaluation of results, Incomplete outcome data, selective result reporting, and other biases. The quality of each trial is divided into "low", "high" or "unclear" risk of bias. When there are different opinions, the two reviewers can discuss or seek third-party consultation to reach a consistent conclusion.

Strategy of data synthesis: We will use Review Manager software version 5.3 provided by Cochrane Collaboration to analyze the data. Dichotomous data is represented by $95 \% \mathrm{RR}$, and continuous data is represented by MD or SMD. When 12 0.01 indicates that the study has no statistical heterogeneity, data will be synthesized using a fixed-effect model; on the contrary, when $12 \geq 50 \%, P<0.01$, indicating the existence of considerrable heterogeneity, the random effects model will be used for analysis[13]. In addition, based on the different causes of heterogeneity, we will further conduct subgroup or sensitivity analysis to find potential causes. If meta-analysis cannot be performed, we will conduct a general descriptive analysis.

Subgroup analysis: We will divide patients diagnosed with COVID-19 into experimental group and control group according to whether they have diabetes, and then conduct subgroup analysis based on different reasons such as age, gender, different forms of intervention, treatment process, drug dosage, etc.

Sensibility analysis: In order to evaluate the robustness of the primary outcome measures, we will eliminate the low-quality studies and combine the data to assess the impact of the sample size, study quality, statistical methods and missing data on the meta-analysis results.

Country(ies) involved: No country restrictions.

Keywords: COVID-19, diabetes mellitus, chloroquine, hydroxychloroquine, metaanalysis, protocol, systematic review.

Contributions of each author:

Author 1 - Yan Liu.

Author 2 - Xiaoxu Fu.

Author 3 - Chunguang Xie. 